CLINICAL STUDY

\title{
Small for gestational age status is associated with metabolic syndrome in overweight children
}

\author{
Thomas Reinehr, Michaela Kleber and Andre Michael Toschke ${ }^{1}$ \\ Department of Pediatric Nutrition Medicine, Vestische Hospital for Children and Adolescents, University of Witten/Herdecke, Dr F Steiner Strasse 5 , \\ 45711 Datteln, Germany and ${ }^{1}$ Division of Health and Social Care Research, King's College London, London, UK \\ (Correspondence should be addressed to T Reinehr; Email: t.reinehr@kinderklinik-datteln.de)
}

\begin{abstract}
Objective: Small for gestational age (SGA) children are at risk of both later obesity and metabolic syndrome (MetS). However, it is unknown whether obesity or SGA status leads to MetS in these subjects. We hypothesized that overweight children with former SGA status had more present components of the MetS than overweight children with former appropriate for gestational age (AGA) status.

Methods: We analyzed 803 overweight children (4\% SGA, mean age $11 \pm 0.1$ years, body mass index (BMI) 27.3 \pm 0.2 , SDS-BMI 2.32 \pm 0.02 ) concerning blood pressure, lipids, glucose, and insulin. Oral glucose tolerance tests (oGTT) were performed in all 35 former SGA children and 147 randomly chosen former non-SGA children.

Results: After adjustment for age, sex, pubertal stage, and BMI-SDS, former SGA status was significantly related to blood pressure, triglyceride, insulin, and $2 \mathrm{~h}$ glucose levels in oGTT. The MetS prevalence was more than doubled in overweight former SGA subjects (40\% MetS) compared with overweight former AGA subjects (17\% MetS). The corresponding adjusted odds ratio was 4.08 (95\% confidence interval 1.48 to 11.22) for SGA compared with AGA children.

Conclusions: Overweight former SGA children had an increased risk for the components of the MetS compared with overweight former AGA children. Therefore, SGA status seems to be a risk factor for the MetS independently of weight status. Particularly overweight children with former SGA status should be screened for the MetS.
\end{abstract}

European Journal of Endocrinology 160 579-584

\section{Introduction}

The clustering of hypertension, dyslipidemia, and disturbed glucose metabolism has been summarized as the metabolic syndrome (MetS) (1) that is associated with atherosclerosis and an increased risk of cardiovascular disease (CVD) $(2,3)$. Additionally, hyperuricemia and non-alcoholic fatty liver disease (NAFLD) are frequent comorbidities of the MetS. Insulin resistance is the postulated underlying mechanism of the MetS (1-3).

During the last decade, many components of the MetS such as high blood pressure, dyslipidemia, glucose intolerance, hyperinsulinism, and type 2 diabetes as well as increased cardiovascular mortality, were observed in adults born small for gestational age (SGA) $(4,5)$. Some of the initial observations in adults have been replicated in children $(6,7)$.

In particular, individuals born SGA with catch-up growth had an increased risk for later CVD and disturbed glucose metabolism (8, 9). Additionally, postnatal accelerated growth and rapid weight gain in
SGA infants were associated with later obesity $(8,9)$. Since obesity per se is associated with components of the MetS such as hypertension, dyslipidemia, and impaired glucose metabolism $(1,10)$, the observed relationship between SGA status at birth and later MetS as a precursor of CVD may be further modified by obesity. If SGA status has a direct impact on insulin resistance as postulated $(11,12)$, overweight children with former SGA status might further deteriorate the cardiovascular risk factor profile as compared with overweight children with former adequate for gestational age (AGA) or large for gestational age (LGA).

To our best knowledge, we are not aware of research comparing cardiovascular risk factors between overweight children with former SGA status and overweight children with former AGA or LGA status. Therefore, we studied 803 overweight children to assess an association between SGA status and components of the MetS. We hypothesized that overweight children with former SGA status had a more unfavorable profile of cardiovascular risk factors compared with overweight children with former AGA or LGA status. 


\section{Methods}

The local ethics committee of the University of Witten/Herdecke approved this study. Written informed consent was obtained from all subjects and their parents prior to study start.

We examined 803 Caucasian overweight children and adolescents aged 4-16 years attending our outpatient center specializing in pediatric obesity following a standardized diagnostic procedure based on the guidelines of the American Obesity Associations (13). All children were screened for degree of overweight, hypertension, dyslipidemia, and disturbed glucose metabolism, as recommended and described below. Exclusion criteria were evidenced for syndromal, chromosomal, or infectious etiology of low birth weight; endocrine or syndromal disorders, systemic disease or acute illness.

Former SGA status was defined by a birth weight or length below the 10th percentile for gestational age using German birth weight and length standards, former LGA as birth weight or length above the 90th percentile for gestational age, and the remaining children as former AGA.

Degree of overweight was derived from body mass index (BMI). Height was measured to the nearest centimeter using a rigid stadiometer. Weight was measured in underwear to the nearest $0.1 \mathrm{~kg}$ using a calibrated balance scale. Overweight was defined by a BMI above the 90th percentile for German children according to the International Task Force for Childhood Obesity $(14,15)$. Because the distribution of BMI is not comparable in children and adults, not even among the various childhood age groups, we used the LMS method to calculate SDS of BMI as a measurement for the degree of overweight. The LMS method summarizes the data in terms of three smooth age-specific curves called $L$ (lambda), $M$ (mu), and $S$ (sigma) based on German population-specific data (16). The $M$ and $S$ curves correspond to the median and coefficient of variation (CV) of BMI for German children at each age and gender, whereas the $L$ curve allows for the substantial agedependent skewness in the distribution of BMI (16). The assumption underlying the LMS method is that after Box-Cox power transformation, the data at each age are normally distributed (16). Height-SDS and weight-SDS were calculated based on German percentiles (15).

Waist and hip circumference were measured as described previously (17). Pubertal stage was determined by well-trained physicians according to Marshall and Tanner. Pubertal developmental stage was categorized into two groups based on breast and genital stages (prepubertal: boys with genital stage I, girls with breast stage I, pubertal: boys with genital stage $>$ II; girls with breast stage $>$ II).

Blood pressure was measured using a validated protocol (18); systolic (SBP) and diastolic (DBP) blood pressure were measured at the right arm twice after a
10 minute rest in the supine position by using a calibrated sphygmomanometer and averaged. The cuff size was based on the length and circumference of the upper arm and was as large as possible without having the elbow skin crease obstructing the stethoscope. The intra- and inter-operator variability was < $5 \%$ for SBP and DBP. Hypertension was defined by blood pressure above the 95th percentile for height, age, and gender (18).

Blood sampling was performed in the fasting status. Serum triglyceride, HDL-cholesterol, uric acid, aspartate transaminase (AST), alanine transaminase (ALT), insulin, and glucose concentrations were measured in all children using commercially available test kits (HDLC Plus, Roche Diagnostics; ALTL-, ASTPL- Cobas Integra 400, Roche Diagnostics; Vitros analyzer Ortho Clinical Diagnostics, Neckargemuend, Germany; MEIA, Abbott). Insulin concentrations were measured by microparticle enhanced immunometric assay (MEIA, Abbott). Intra- and interassay variations for the concentrations $(\mathrm{CV})$ of these variables were less than $5 \%$. Homeostasis model assessment (HOMA) as an assessment of insulin resistance was calculated as follows (19): resistance $(\mathrm{HOMA})=($ insulin $(\mathrm{mU} / \mathrm{l}) \times$ glucose $(\mathrm{mmol} / \mathrm{l})) / 22.5$. Impaired fasting glucose was defined by $>5.5 \mathrm{mmol} / \mathrm{l}(100 \mathrm{mg} / \mathrm{dl})(20)$.

An oral glucose tolerance test (oGTT) was performed in all SGA children and in a random sample of 147 AGA and 8 LGA children according to guidelines (21). Impaired glucose tolerance was defined by $2 \mathrm{~h}$ serum glucose $>140 \mathrm{mg} / \mathrm{dl}$ in the oGTT. In children with oGTT, we defined the MetS according to the definition of Weiss (22) (BMI >97th percentile plus 3 of the following criteria: blood pressure $>95$ th percentile for height, age and gender (18); triglycerides >95th percentile (23); HDL < 5 th percentile (23); impaired glucose tolerance). We used this definition for the MetS, since intima-media thickness as a predictive marker for atherosclerosis and later cardiovascular events $(24,25)$ correlated more strongly to the definition of Weiss compared with any other proposed definition of the MetS in children and adolescents (25).

\section{Statistical analysis}

Mean values and corresponding confidence intervals (CI) of continuous variables were calculated based on the normal distribution and for binary variables based on the binomial distribution. Comparisons of study characteristics were carried out with ANOVA or $\chi^{2}$ tests respectively. Comparisons of mean values of continuous variables by size status at birth (SGA, AGA or LGA) used dummy variables and were carried out using separate multivariable linear regression analyses adjusting for age, gender, pubertal stage, and degree of overweight. Multivariable logistic regression was used for binary outcomes and adjusted for the same confounders as the linear analysis. 
Table 1 Study characteristics of small for gestational age (SGA), appropriate for gestational age (AGA), large for gestational age (LGA) children.

\begin{tabular}{|c|c|c|c|c|c|}
\hline \multirow[b]{2}{*}{ Variable } & \multicolumn{4}{|c|}{ Mean (S.E.M.) } & \multirow[b]{2}{*}{$P$ value } \\
\hline & SGA $(n=35)$ & AGA $(n=726)$ & LGA $(n=42)$ & Total $(n=803)$ & \\
\hline Age (years) & $10(0.4)$ & $11(0.1)$ & $10(0.3)$ & $10.9(0.1)$ & 0.008 \\
\hline Male sex (\%) & $31(7.8)$ & $43(1.8)$ & $69(7.1)$ & $43(1.7)$ & 0.001 \\
\hline Prepubertal stage (\%) & $54(8.4)$ & $47(1.9)$ & $56(7.7)$ & $48(1.8)$ & 0.071 \\
\hline Height $(\mathrm{cm})$ & $145.5(2.3)$ & $152.4(0.6)$ & $152.4(2.1)$ & $152.1(0.6)$ & 0.037 \\
\hline SDS-height & $0.28(0.16)$ & $0.39(0.04)$ & $1.09(0.14)$ & $0.42(0.04)$ & $<0.001$ \\
\hline Weight $(\mathrm{kg})$ & $57.4(2.9)$ & $65.1(0.7)$ & $66.5(3.2)$ & $64.8(0.7)$ & 0.068 \\
\hline SDS-weight & $2.05(0.11)$ & $2.06(0.03)$ & $2.46(0.10)$ & $2.08(0.03)$ & 0.002 \\
\hline BMI $\left(\mathrm{kg} / \mathrm{m}^{2}\right)$ & $26.4(0.7)$ & $27.3(0.2)$ & $28.0(0.7)$ & $27.3(0.15)$ & 0.279 \\
\hline SDS-BMI & $2.39(0.08)$ & $2.31(0.02)$ & $2.53(0.08)$ & $2.32(0.02)$ & 0.013 \\
\hline Birth weight (g) & $2474(77)$ & 3489 (19) & $4550(84)$ & $3500(21)$ & $<0.001$ \\
\hline Birth length $(\mathrm{cm})$ & $47(0.6)$ & $51(0.1)$ & $53(0.3)$ & $51(0.1)$ & $<0.001$ \\
\hline Gestational age (weeks) & $39(0.5)$ & $39(0.1)$ & $39(0.3)$ & $39(0.1)$ & 0.108 \\
\hline
\end{tabular}

All calculations were carried out with the software package SAS version 9.1.3 (SAS Institute Inc., Cary, NC, USA).

\section{Results}

A total of $35(4 \%)$ children of the examined 803 children had a former SGA status, 5\% were LGA, and $91 \%$ of the children were AGA. The study characteristics are shown in Table 1. All infants had a gestational age between 34 and 42 weeks. Twentyeight $(80 \%)$ of the children with former SGA status were proportional at birth (birth height and birth weight $<10$ th percentile) and seven $(20 \%)$ of children with the former SGA were disproportional (birth height $<10$ th percentile and birth weight $>10$ th percentile or vice versa). No differences in gestational age could be observed between SGA, AGA, and LGA children in the entire study sample. SGA children had on average a lower height ( $P=0.037$ and $P<0.001$ for SDS-height). LGA children had the highest BMI-SDS values $(P=0.013)$. AGA, SGA, and LGA children did not differ significantly in pubertal stage. There was also no significant difference in pubertal stage, when analyzing only female subjects $(P=0.108)$.

In separate multivariable linear regression models considering size at birth in three categories (SGA, AGA, LGA) and adjusting for age, sex, pubertal stage, and BMI-SDS, former SGA status was significantly related to blood pressure, triglyceride, insulin, and $2 \mathrm{~h}$ glucose levels in the oGTT (Table 2). Uric acids and HOMA levels were borderline increased in SGA children $(P=0.054$ and $P=0.080)$. Mean levels of fasting glucose did not differ between SGA, LGA, and AGA children $(P=0.947)$. ALT levels were significantly higher in former SGA and LGA children compared with AGA children. HDL cholesterol levels and waist circumference did not differ between SGA, AGA, and LGA children $(P=0.879$ and 0.299$)$.

Table 2 Mean levels of metabolic parameters among 803 children and adolescents.

\begin{tabular}{llllr}
\hline & \multicolumn{3}{c}{ Mean (s.E.M.) } & Exact adjusted $\boldsymbol{P}$ value \\
\cline { 2 - 4 } Variable & SGA $(n=35)$ & AGA $(n=726)$ & LGA $(n=42)$ & 0.812 \\
\hline Waist-to-hip ratio & $0.96(0.002)$ & $0.97(0.005)$ & $0.97(0.002)$ & 0.299 \\
Waist circumference $(\mathrm{cm})$ & $86(2.6)$ & $91(0.7)$ & $94(3.5)$ & 0.012 \\
Systolic blood pressure $(\mathrm{mmHg})$ & $120(2.4)$ & $115(0.5)$ & $113(1.8)$ & $<0.001$ \\
Diastolic blood pressure (mmHg) & $70(2.2)$ & $64(0.4)$ & $63(1.7)$ & 0.947 \\
Fasting glucose (mmol/l) & $4.7(0.1)$ & $4.7(0.02)$ & $4.7(0.1)$ & 0.004 \\
2 h glucose in oGTT (mmol/l) & $7.6(0.1)$ & $6.8(0.1)$ & $7.1(0.4)$ & 0.879 \\
HDL-cholesterol (mmol/l) & $1.27(0.04)$ & $1.31(0.01)$ & $1.29(0.04)$ & 0.032 \\
Triglycerides (mmol/l) & $1.35(0.12)$ & $1.20(0.02)$ & $1.46(0.13)$ & 0.033 \\
Insulin (mU/l) & $19.7(1.4)$ & $16.8(0.5)$ & $14.9(1.3)$ & 0.080 \\
HOMA & $4.1(0.3)$ & $3.6(0.1)$ & $3.2(0.3)$ & 0.627 \\
AST $(\mathrm{U} / \mathrm{l})$ & $24(1.9)$ & $24(0.4)$ & $26(1.6)$ & $<0.001$ \\
ALT $(\mathrm{U} / \mathrm{l})$ & $32(4.5)$ & $26(0.6)$ & $36(6.1)$ & 0.054 \\
Uric acid $(\mu \mathrm{mol} / \mathrm{l})$ & $285(12)$ & $279(2)$ & $267(12)$ &
\end{tabular}

oGTT, oral glucose tolerance test.

${ }^{a}$ Derived from multivariable linear regression considering size status at birth in 3 categories (SGA, AGA, LGA) and adjusted for age, sex, SDS-BMI, and pubertal stage; conversion factors: glucose: $\mathrm{mg} / \mathrm{dl}=\mathrm{mmol} / \mathrm{l} \times 18$; $\mathrm{HDL}$-cholesterol: $\mathrm{mg} / \mathrm{dl}=\mathrm{mmol} / \mathrm{l} \times 38.7$; triglyceride: $\mathrm{mg} / \mathrm{dl}=\mathrm{mmol} / \mathrm{l} \times 88.57$; uric acid: $\mathrm{mg} / \mathrm{dl}=\mu \mathrm{mol} / \mathrm{l} \times 0.017$.

$\mathrm{b}_{n=1} 190$. 
In summary, all components considered in MetS definitions except low HDL-cholesterol levels were deteriorated in SGA children compared with AGA children and the proportion of hypertension, hypertriglyceridaemia, glucose intolerance, and MetS was significantly higher in SGA children compared with AGA and LGA children in separate multivariable logistic regression models considering size at birth in three categories (SGA, AGA, LGA; Table 3). The MetS prevalence was more than doubled in SGA subjects compared with AGA subjects. The corresponding adjusted odds ratio was 4.08 (95\% CI 1.48 to 11.22 ) for SGA children compared with AGA children.

Sensitivity analyses focused on SGA and AGA children and considered the association of birth weight as an explanatory variable. Univariate analysis did not show significant associations between birth weight and cardiovascular risk factors (all $P>0.05$ ) except for impaired glucose tolerance $(P=0.003)$. However, after adjustment for gestational age the analysis yielded similar results with respect to cardiovascular risk factors compared with the analysis considering the categorical SGA variable. However, triglyceride levels were not associated with birth weight adjusted for gestational age. Birth length adjusted for gestational age was not associated with any of the cardiovascular risk factors.

\section{Discussion}

This is the first study in overweight children analyzing the impact of former SGA status on the prevalence of the MetS and its components. We observed an increase of cardiovascular risk factors in overweight children with former SGA status in terms of higher frequency of hypertension, hypertriglyceridaemia, impaired glucose tolerance, and consequently the prevalence of MetS as compared with overweight children with former AGA

Table 3 Prevalence of metabolic syndrome and its components among 803 children and adolescents.

\begin{tabular}{|c|c|c|c|c|}
\hline \multirow[b]{2}{*}{ Variable } & \multicolumn{3}{|c|}{ Prevalence (S.E.M.) } & \multirow{2}{*}{$\begin{array}{c}\text { Exact } \\
\text { adjusted } \\
P \text { value }\end{array}$} \\
\hline & $\begin{array}{c}\text { SGA } \\
(n=35)\end{array}$ & $\begin{array}{c}\text { AGA } \\
(n=726)\end{array}$ & $\begin{array}{c}\text { LGA } \\
(n=42)\end{array}$ & \\
\hline Hypertension & $43(8.4)$ & $24(1.6)$ & $21(6.3)$ & 0.032 \\
\hline $\begin{array}{l}\text { Triglycerides } \\
>95 \text { th percentile }\end{array}$ & $26(7.4)$ & 15 (1.3) & $31(7.1)$ & 0.024 \\
\hline $\begin{array}{l}\text { HDL-cholesterol } \\
<5 \text { th percentile }\end{array}$ & $14(5.9)$ & $16(1.4)$ & $15(5.5)$ & 0.962 \\
\hline $\begin{array}{l}\text { Impaired glucose } \\
\text { tolerance }^{b}\end{array}$ & $51(8.4)$ & $18(3.2)$ & $25(6.7)$ & $<0.001$ \\
\hline $\begin{array}{l}\text { Metabolic } \\
\text { syndrome }^{\text {b }}\end{array}$ & $40(8.3)$ & $17(3.1)$ & $25(6.7)$ & 0.018 \\
\hline
\end{tabular}

${ }^{a}$ Derived from multivariable logistic regression considering size status at birth in three categories (SGA, AGA, LGA) and adjusted for age, sex, SDS-BMI, and pubertal stage.

$\mathrm{b}_{n=190 .}$ status. Furthermore, we observed factors associated with the MetS such as hyperuricemia and increased ALT levels as an indirect hint for NAFLD among children with former SGA status more frequent than among children with former AGA status. These findings suggest that SGA status is a risk factor for the MetS independently of weight status.

The association between SGA status and hypertriglyceridemia, hypertension, and impaired glucose metabolism is in concordance with previous studies (7, 26-28). However, waist circumference and HDL cholesterol levels as major components of the MetS were not related to SGA status in our study of overweight children. Low HDL cholesterol levels were predominantly observed among overweight adolescents and not in younger children $(29,30)$, and might explain the lack of association between HDL cholesterol and SGA status in the present study due to an age of below 14 years in the great majority of the considered children. Values of waist circumference depend on age and gender and are only a weak measurement of abdominal fat mass possibly explaining the missing link between SGA status and waist circumference.

The former SGA children were shorter than AGA and LGA children. However, the mean SDS-height was greater than zero in the overweight children with former SGA status suggesting that these children have had an accelerated growth at some point after term. This finding is in accordance with the increased prevalence of obesity among SGA infants with catchup growth (8). In most SGA children, catch-up was largely completed by 2 years of age (31). By contrast, other studies have reported that gain in BMI during early childhood, but after infancy, is an important factor for both, body fat mass and sequelae $(32,33)$.

One major consequence of early accelerated growth and rapid postnatal weight gain in SGA children seems to be a deterioration of glucose and insulin metabolism resulting in insulin resistance, which is the postulated underlying mechanism of MetS even in children $(1,34)$. Barker postulated that the postnatal increase in insulin resistance in SGA children may result from the fetal adaptation to an adverse intrauterine environment during a critical time period, leading to a long-lasting programming of fetal gene expression (5). Several underlying mechanisms have been suggested (35), each of them point to a detrimental fetal environment, a genetic susceptibility or an interaction between these two components. Insulin secreted by the fetal pancreas in response to maternal glucose concentrations is a suggested key growth factor. Monogenic diseases that impair sensing of glucose, lower insulin secretion or increased insulin resistance are associated with impaired fetal growth. Polygenic influences resulting in insulin resistance in the normal population are therefore likely to result in lower birth weight. Abnormal vascular development during fetal life and early childhood, as a result of genetic insulin resistance, 
could also explain the increased risk of hypertension and vascular disease.

The extent of postnatal weight gain in SGA children contributes not only to adult size and body proportions but also has been linked to changes in insulin sensitivity $(36,37)$. By contrast, glucose and insulin levels were stronger related to childhood size than size at birth in 10-11-year-old children (38). However in this study, size at birth was not adjusted for gestational age. In our study, birth weight not adjusted to gestational age was also not related to insulin, glucose or insulin resistance index HOMA or any components of the MetS, while former SGA status was related to insulin levels, $2 \mathrm{~h}$ glucose levels in oGTT and borderline in insulin resistance index HOMA. These findings suggest that SGA status with rapid postnatal weight gain is likely to be associated with impairment in insulin sensitivity. Nonetheless, the relative contribution of low birth weight, accelerated postnatal growth, and weight gain to the development of insulin resistance is not known.

By contrast to former SGA status, LGA status at birth was not associated to components of the MetS. However, former LGA children had a significantly higher BMI-SDS than former SGA and AGA children in this study. This finding is in line with the theory that SGA status and the associated insulin resistance is the underlying mechanism of components of the MetS and not the degree of overweight.

This study has some potential important limitations. First, we did not have exact information concerning the etiology of SGA status. For example, the effect of SGA status on cardiovascular risk factors may be different in children of mothers with pre-eclampsia, placental insufficiency or mothers who had smoked during pregnancy. Secondly, the time and degree of postnatal rapid weight gain and accelerated growth in children with former SGA status was unknown, possibly also modifying the prevalence of the MetS and its components. Thirdly, data from clinical samples may not be representative for general populations and selection and referral bias may have influenced our estimates of the prevalence of the MS. However, the distribution of lipids, glucose, and blood pressure values in our study were similar to those reported from larger population based studies or studies including different ethnicities (39). Furthermore, different ethnicities have to be studied in particular, since for instance waist circumference depends on race (40). Fourthly, BMI percentiles were used to classify overweight. Although BMI is a good measure for overweight, it has limitations as an indirect measure of fat mass. Fifthly, HOMA is only an assessment of insulin sensitivity and clamp studies are the gold standard to analyze insulin resistance (41). Sixth, prepubertal and pubertal children, as well as boys and girls with former SGA status were analyzed together in this study due to the limited number of children with former SGA status. Probably, both gender and pubertal status influence the prevalence of the MetS and cardiovascular risk factors in overweight children with former SGA. However, the multivariable analyses performed in this study considered both gender and pubertal stage and thus should allow for appropriately adjusted estimates. Further larger analyses are necessary to analyze these potential influence factors. Finally, this is a cross-sectional study that cannot prove causality. Longitudinal studies analyzing the onset of components of the MetS in SGA children are required to identify the optimal screening point for CVDs.

In summary, overweight children with former SGA status had an increased risk for hypertension, hypertriglyceridemia, impaired glucose tolerance, and a more than doubled risk for the MetS as compared with overweight children with former AGA status. Therefore, SGA status seems to be a risk factor for the MetS independently of weight status. In conclusion, overweight children with former SGA status should be screened for components of the MetS.

\section{Declaration of interest}

The authors declare that there is no conflict of interest prejudicing the impartiality of the scientific work reported.

\section{Funding}

This study is registered at clinicaltrials.gov (NCT00435734). This study was funded in part by NovoNordisk Pharma GmbH Mainz, Germany and by a grant support from of the German 'Competence Net Obesity,' which is supported by the German Federal Ministry of Education and Research (grant number 01 GI0839).

\section{References}

1 Reaven GM. Insulin resistance and compensatory hyperinsulinemia: role in hypertension, dyslipidemia, and coronary heart disease. American Heart Journal 1991121 1283-1288.

2 Eckel RH, Grundy SM \& Zimmet PZ. The metabolic syndrome. Lancet $20053651415-1428$.

3 Isomaa B, Almgren P, Tuomi T, Forsen B, Lahti K, Nissen M, Taskinen MR \& Groop L. Cardiovascular morbidity and mortality associated with the metabolic syndrome. Diabetes Care 200124 683-689.

4 Barker DJ, Hales CN, Fall CH, Osmond C, Phipps K \& Clark PM. Type 2 (non-insulin-dependent) diabetes mellitus, hypertension and hyperlipidaemia (syndrome $\mathrm{X}$ ): relation to reduced fetal growth. Diabetologia 199336 62-67.

5 Barker DJ. Fetal origins of coronary heart disease. BMJ 1995311 171-174.

6 Whincup P, Cook D, Papacosta O \& Walker M. Birth weight and blood pressure: cross sectional and longitudinal relations in childhood. BMJ 1995311 773-776.

7 Tenhola S, Martikainen A, Rahiala E, Herrgard E, Halonen P \& Voutilainen R. Serum lipid concentrations and growth characteristics in 12-year-old children born small for gestational age. Pediatric Research 200048 623-628.

8 Ong KK, Ahmed ML, Emmett PM, Preece MA \& Dunger DB. Association between postnatal catch-up growth and obesity in childhood: prospective cohort study. BMJ 2000320 967-971.

9 Crowther NJ, Cameron N, Trusler J \& Gray IP. Association between poor glucose tolerance and rapid post natal weight gain in sevenyear-old children. Diabetologia 199841 1163-1167. 
10 Ebbeling CB, Pawlak DB \& Ludwig DS. Childhood obesity: publichealth crisis, common sense cure. Lancet $2002360473-482$.

11 Ibanez L, Ong K, Dunger DB \& de ZF. Early development of adiposity and insulin resistance after catch-up weight gain in small-for-gestational-age children. Journal of Clinical Endocrinology and Metabolism 200691 2153-2158.

12 Soto N, Bazaes RA, Pena V, Salazar T, Avila A, Iniguez G, Ong KK, Dunger DB \& Mericq MV. Insulin sensitivity and secretion are related to catch-up growth in small-for-gestational-age infants at age 1 year: results from a prospective cohort. Journal of Clinical Endocrinology and Metabolism 200388 3645-3650.

13 Barlow SE. Expert committee recommendations regarding the prevention, assessment, and treatment of child and adolescent overweight and obesity: summary report. Pediatrics $2007 \mathbf{1 2 0}$ S164-S192.

14 Cole TJ, Bellizzi MC, Flegal KM \& Dietz WH. Establishing a standard definition for child overweight and obesity worldwide: international survey. BMI 2000320 1240-1243.

15 Kromeyer-Hauschild K, Wabitsch M, Geller F, Ziegler A, Geiss HC, Hesse V, von Hippel V, Jäger U, Johnson D, Korte W, Kunze D, Menner K, Müller G, Müller M, Niemann-Pilatus A, Remer T, Schäfer F, Wittchen H, Zabransky S, Zelnner K \& Hebebrand J. Percentiles of body mass index in children and adolescents evaluated from different regional German studies. Monatsschrift für Kinderheilkunde 2001149 807-818.

16 Cole TJ. The LMS method for constructing normalized growth standards. European Journal of Clinical Nutrition 1990 44 45-60.

17 Kromeyer-Hausschild K, Gläßer N \& Zellner K. Waist circumference percentile in Jena children (Germany) 6 to 18 years of age. Aktuelle Ernährungsmedizin 200833 116-122.

18 National High Blood Pressure Education Program. The fourth report on the diagnosis, evaluation, and treatment of high blood pressure in children and adolescents. Pediatrics $2004 \mathbf{1 1 4}$ $555-576$.

19 Matthews DR, Hosker JP, Rudenski AS, Naylor BA, Treacher DF \& Turner RC. Homeostasis model assessment: insulin resistance and beta-cell function from fasting plasma glucose and insulin concentrations in man. Diabetologia 198528 412-419.

20 Genuth S, Alberti KG, Bennett P, Buse J, Defronzo R, Kahn R, Kitzmiller J, Knowler WC, Lebovitz H, Lernmark A, Nathan D, Palmer J, Rizza R, Saudek C, Shaw J, Steffes M, Stern M, Tuomilehto J \& Zimmet P. Follow-up report on the diagnosis of diabetes mellitus. Diabetes Care 200326 3160-3167.

21 American Diabetes Association. Diagnosis and classification of diabetes mellitus. Diabetes Care 200831 S55-S60.

22 Weiss R, Dziura J, Burgert TS, Tamborlane WV, Taksali SE, Yeckel CW, Allen K, Lopes M, Savoye M, Morrison J, Sherwin RS \& Caprio S. Obesity and the metabolic syndrome in children and adolescents. New England Journal of Medicine 2004350 2362-2374.

23 http:www.a-g-a.de/Leitlinie.pdf. Guidelines of the German working group on obese children and adolescents, 2008.

24 Reinehr T, Kiess W, de SG, Stoffel-Wagner B \& Wunsch R. Intima media thickness in childhood obesity: relations to inflammatory marker, glucose metabolism, and blood pressure. Metabolism 2006 55 113-118.

25 Reinehr T, Wunsch R, de SG \& Toschke AM. Relationship between metabolic syndrome definitions for children and adolescents and intima-media thickness. Atherosclerosis 2008199 193-200.

26 Donker GA, Labarthe DR, Harrist RB, Selwyn BJ, Srinivasan SR, Wattigney W \& Berenson GS. Low birth weight and serum lipid concentrations at age 7-11 years in a biracial sample. American Journal of Epidemiology $1997 \mathbf{1 4 5}$ 398-407.
27 Donker GA, Labarthe DR, Harrist RB, Selwyn BJ, Wattigney W \& Berenson GS. Low birth weight and blood pressure at age 7-11 years in a biracial sample. American Journal of Epidemiology 1997 145 387-397.

28 Rich-Edwards JW, Kleinman K, Michels KB, Stampfer MJ, Manson JE, Rexrode KM, Hibert EN \& Willett WC. Longitudinal study of birth weight and adult body mass index in predicting risk of coronary heart disease and stroke in women. BMJ 2005330 1115.

29 I'Allemand D, Wiegand S, Reinehr T, Muller J, Wabitsch M, Widhalm K \& Holl R. Cardiovascular risk in 26,008 European overweight children as established by a multicenter database. Obesity 200816 1672-1679.

30 Reinehr T, Andler W, Denzer C, Siegried W, Mayer H \& Wabitsch M. Cardiovascular risk factors in overweight German children and adolescents: relation to gender, age and degree of overweight. Nutrition, Metabolism, and Cardiovascular Diseases 200515 181-187.

31 Hokken-Koelega AC, De Ridder MA, Lemmen RJ, Den HH, De Muinck Keizer-Schrama SM \& Drop SL. Children born small for gestational age: do they catch up? Pediatric Research $1995 \mathbf{3 8}$ $267-271$.

32 Bhargava SK, Sachdev HS, Fall CH, Osmond C, Lakshmy R, Barker DJ, Biswas SK, Ramji S, Prabhakaran D \& Reddy KS. Relation of serial changes in childhood body-mass index to impaired glucose tolerance in young adulthood. New England Journal of Medicine 2004350 865-875.

33 Toschke AM, Ruckinger S, Reinehr T \& von KR. Growth around puberty as predictor of adult obesity. European Journal of Clinical Nutrition 200862 1405-1411.

34 Reinehr T, de SG \& Andler W. Longitudinal analyses among overweight, insulin resistance, and cardiovascular risk factors in children. Obesity Research 200513 1824-1833.

35 Levy-Marchal C \& Czernichow P. Small for gestational age and the metabolic syndrome: which mechanism is suggested by epidemiological and clinical studies? Hormone Research 200665 123-130.

36 Ibanez L, Suarez L, Lopez-Bermejo A, Diaz M, Valls C \& de ZF. Early development of visceral fat excess after spontaneous catch-up growth in children with low birth weight. Journal of Clinical Endocrinology and Metabolism 200893 925-928.

37 Veening MA, van Weissenbruch MM \& Delemarre-van de Waal HA. Glucose tolerance, insulin sensitivity, and insulin secretion in children born small for gestational age. Journal of Clinical Endocrinology and Metabolism 200287 4657-4661.

38 Whincup PH, Cook DG, Adshead F, Taylor SJ, Walker M, Papacosta $O$ \& Alberti KG. Childhood size is more strongly related than size at birth to glucose and insulin levels in 10-11-year-old children. Diabetologia 199740 319-326.

39 Csabi G, Torok K, Jeges S \& Molnar D. Presence of metabolic cardiovascular syndrome in obese children. European Journal of Pediatrics 2000159 91-94.

40 Cruz ML, Weigensberg MJ, Huang TT, Ball G, Shaibi GQ \& Goran MI. The metabolic syndrome in overweight Hispanic youth and the role of insulin sensitivity. Journal of Clinical Endocrinology and Metabolism 200489 108-113.

41 Wallace TM \& Matthews DR. The assessment of insulin resistance in man. Diabetic Medicine 200219 527-534.

Received 13 January 2009

Accepted 14 January 2009 